\title{
VDAC1-based peptides: novel pro-apoptotic agents and potential therapeutics for B-cell chronic lymphocytic leukemia
}

\author{
T Prezma ${ }^{1,3}$, A Shteinfer ${ }^{1,3}$, L Admoni ${ }^{1}$, Z Raviv $^{1}$, I Sela $^{1}$, I Levi $^{2}$ and V Shoshan-Barmatz ${ }^{*, 1}$
}

The voltage-dependent anion channel 1 (VDAC1), localized in the outer mitochondrial membrane, mediates metabolic cross-talk between the mitochondrion and the cytoplasm and thus serves a fundamental role in cell energy metabolism. VDAC1 also plays a key role in mitochondria-mediated apoptosis, interacting with anti-apoptotic proteins. Resistance of cancer cells to apoptosis involves quenching the mitochondrial apoptotic pathway by over-expression of anti-apoptotic/pro-survival hexokinase (HK) and $\mathrm{Bcl}-2$ family proteins, proteins that mediate their anti-apoptotic activities via interaction with VDAC1. Using specifically designed VDAC1-based cell-penetrating peptides, we targeted these anti-apoptotic proteins to prevent their pro-survival/anti-apoptotic activities. Anti-apoptotic proteins are expressed at high levels in B-cell chronic lymphocytic leukemia (CLL), an incurable disease requiring innovative new approaches to improve therapeutic outcome. CLL is characterized by a clonal accumulation of mature neoplastic B cells that are resistant to apoptosis. Specifically, we demonstrate that the VDAC1-based peptides (Antp-LP4 and N-Terminal-Antp) selectively kill peripheral blood mononuclear cells (PBMCs) obtained from CLL patients, yet spare those obtained from healthy donors. The cell death induction competence of the peptides was well correlated with the amount of double positive CD19/CD5 cancerous CLL PBMCs, further illustrating their selectivity toward cancer cells. Moreover, these VDAC1-based peptides induced apoptosis by activating the mitochondria-mediated pathway, reflected in membrane blebbing, condensation of nuclei, DNA fragmentation, release of mitochondrial cytochrome $c$, loss of mitochondrial membrane potential, decreased cellular ATP levels and detachment of HK, all leading to apoptotic cell death. Thus, the mode of action of the peptides involves decreasing energy production and inducing apoptosis. Over 27 versions of cell-penetrating VDAC1-based peptides were designed and screened to identify the most stable, short and apoptosis-inducing peptides toward CLL-derived lymphocytes. In this manner, three optimized peptides suitable for in vivo studies were identified. This study thus reveals the potential of VDAC1-based peptides as an innovative and effective anti-CLL therapy.

Cell Death and Disease (2013) 4, e809; doi:10.1038/cddis.2013.316; published online 19 September 2013

Subject Category: Cancer

B-chronic lymphocytic leukemia (CLL) is characterized by the accumulation of CD5 + monoclonal B cells in the blood, bone marrow and peripheral lymphoid organs. ${ }^{1}$ These correspond to mature $B$ cells that have evaded apoptosis and undergone cell-cycle arrest in the G0/G1 phase, thereby possessing low proliferative activity. ${ }^{1}$ Thus, in CLL, in contrast to acute leukemia or aggressive lymphomas, which are characterized by uncontrolled growth and high proliferation, failure to undergo apoptosis constitutes the primary cellular defect. ${ }^{2}$ The anti-apoptotic proteins of the Bcl-2 family, such as Bcl-2, $\mathrm{Bcl}-\mathrm{xL}$, and $\mathrm{Mcl}-1$ and $\mathrm{XIAP}$, are over-expressed in CLL, whereas pro-apoptotic protein Bax is under-expressed., ${ }^{3,4}$ Chemotherapy treatment of CLL usually involves fludarabine, together with alkylating agents, such as cyclophosphamide ${ }^{5}$ and humanized monoclonal antibodies. ${ }^{6}$ Despite improvements in treatment, CLL is still considered incurable.
Even in the presence of oxygen, most cancers, including CLL, rely on glycolysis as the main energy-generating pathway (i.e. the Warburg effect) and as a source of building blocks for proteins, nucleotides and lipids. ${ }^{7,8}$ Such cancerinduced metabolic re-programming also includes overexpression of the mitochondria-bound glycolytic protein, hexokinase (HK). HK acts as an anti-apoptotic protein via direct association with the voltage-dependent anion channel1 (VDAC1). ${ }^{5-7,9}$ VDAC1, localized in the outer mitochondrial membrane, plays a central role in cell metabolism by mediating the transfer of metabolites between the mitochondrion and the cytosol. ${ }^{10}$ Moreover, VDAC1 is a key player in mitochondria-mediated apoptosis, participating in the release of mitochondrial pro-apoptotic proteins to the cytosol (e.g. cytochrome $c$ (Cyto $c$ ), AIF and Smac/DIABLO), ${ }^{5,11,12}$ and interacting with apoptosis regulatory proteins, such as

\footnotetext{
${ }^{1}$ Department of Life Sciences and the National Institute for Biotechnology in the Negev, BeerSheva, Israel and ${ }^{2}$ Department of Hematology, Soroka University Medical Center and the Faculty of Health Sciences, Ben-Gurion University of the Negev, BeerSheva 84105, Israel

${ }^{*}$ Corresponding author: Professor V Shoshan-Barmatz, Department of Life Sciences, Ben-GurionUniversity of the Negev, Building 40, Beer-Sheva 84105, Israel. Tel: + 9728 6461336; Fax: + 9728 6472992; E-mail: vardasb@bgu.ac.il

${ }^{3}$ These authors contributed equally to this work.

Keywords: apoptosis; CLL; metabolism; mitochondria; peptides; VDAC1

Abbreviations: EGS, ethylene glycol-bis(succinimidyl succinate); HK-I, hexokinase-l; PBMCs, peripheral mononuclear cells; VDAC, voltage-dependent anion channel Received 30.4.13; revised 15.7.13; accepted 19.7.13; Edited by M Agostini
} 
$\mathrm{Bcl}-2, \mathrm{Bcl}-\mathrm{xL}^{13-15}$ and $\mathrm{HK},{ }^{12,13,16,17}$ that are over-expressed in many cancers, including CLL. ${ }^{18,19}$ Thus, VDAC1 can control the fate of cancer cells. ${ }^{13,20}$

Via point mutations, we identified VDAC1 domains and amino-acid residues important for interaction with $\mathrm{HK}, \mathrm{Bcl}-2$ and $\mathrm{Bcl}-\mathrm{xL}$ and designed VDAC1-based peptides targeting these interactions, thereby inhibiting the anti-apoptotic effects of these proteins. ${ }^{12-17}$ Antp-LP4 is a cell-penetrating VDAC1based peptide comprising Antp (penetrating), a 16 residuelong sequence from the Drosophila antennapedia-homeodomain, fused to a VDAC1-derived sequence. The AntpLP4 peptide did not induce cell death in normal peripheral blood lymphocytes (PBMCs). ${ }^{17}$

Here, we demonstrate that the VDAC1-based peptides, Antp-LP4 and N-Terminal-Antp (N-Ter-Antp) peptides and improved versions thereof selectively induced cell death of PBMCs from CLL patients yet exhibited only minor effects on PBMCs from healthy donors. The mode of action of the peptides involves dysfunction of mitochondria energy production and apoptosis induction. These results demonstrate the potential of VDAC1-based peptides for treating CLL.

\section{Results}

We previously demonstrated that the cell-penetrating VDAC1based Antp-LP4 peptide diminished the anti-apoptotic effects of HK-I, Bcl-2 or Bcl-xL, ${ }^{14,15,17}$ and induced cell death in several cancer cell lines yet did not harm normal PBMCs. ${ }^{17}$ The loop-shaped Antp-LP4 peptide corresponds to a VDAC1derived sequence (designated LP4) flanked by a tryptophan zipper motif. The SWTWE sequence at the N-terminal and the KWTWK sequence at the C-terminal of the VDAC1-derived peptide, allowing for the formation of a tryptophan zipper and a stable $\beta$-hairpin, ${ }^{21}$ mimics the VDAC1 LP4 loop of origin. The peptide is preceded by the cell-penetrating sequence, Antp (Figure 6a). We designed and tested 27 versions of cellpenetrating VDAC1-based peptides to assess their potential use in CLL therapy.

The VDAC1-based Antp-LP4 peptide induces death of CLL cells. The ability of Antp-LP4 peptide to induce cell death was compared in PBMCs isolated from blood collected from 51 CLL patients not undergoing treatment (Supplementary Table 1) and 34 healthy donors (Figure 1a), and used immediately or cryo-preserved in liquid nitrogen. Incubation of PBMCs for $90 \mathrm{~min}$ with Antp-LP4 resulted in marked cell death of the CLL-derived cells but only slightly affected cells isolated from healthy donors (Figure 1a).

In both CLL-derived PBMCs and the MEC-1 cell line, closely resembling B-CLL, ${ }^{22}$ Antp-LP4 induced cell death in a dose-dependent manner (Figure 1b). The data were fitted to calculate the peptide concentration required for half-maximal cell killing activity $\left(\mathrm{IC}_{50}\right)$. $\mathrm{IC}_{50}$ values for Antp-LP4 of $0.7 \pm 0.1 \mu \mathrm{M}$ and $2.5 \pm 0.1 \mu \mathrm{M}$ in CLL-derived PBMCs and MEC-1 cells, respectively, were obtained (Table 1).

Next, we analyzed the relative amounts of CD5 + /CD19+ cells, representing cancerous cells, in the PBMCs isolated from each CLL patient using specific monoclonal antibodies (Figures 1c and d). Representative flow cytometric analyses of such experiments on PBMCs from a single CLL patient and healthy donor demonstrate that in the CLL patient, about $76 \%$ of the cells are CD5 +/CD19+ (Figure 1d), whereas less than $2 \%$ of such cells are found in healthy donor PBMCs (Figure 1c).

To verify the relationship between the cell killing capacity of Antp-LP4 and cancerous cell amounts, the extent of Antp-LP4-induced cell death was plotted as a function of the percentage of CD5 $+/ C D 19+$ cells for each patient (Figure 1e). The degree of cell death induced by Antp-LP4 rose with the increase in CD5 + /CD19+ levels. This revealed a correlation between the cell killing capacity of the peptide and CD5 + /CD19 + cell amounts, further demonstrating the specificity of Antp-LP4 toward cancerous CLL cells.

Furthermore, cells expressing CD5 + /CD19 + correspond to those undergoing apoptosis, as revealed by staining with 7-amino-actinomycin (7AAD) instead of propidium iodide (PI), performed to overcome any overlap with anti-CD5 + /CD19+ antibody labeling (Figure 1f).

Antp-LP4 decreases the mitochondrial membrane potential and cellular ATP levels in CLL PBMCs. To elucidate the mode of action of Antp-LP4 in inducing cell death, we first examined the effect of the peptide on the mitochondrial membrane potential $(\triangle \Psi \mathrm{m})$. PBMCs were incubated for a short time $(15 \mathrm{~min})$ and with relatively low concentrations of Antp-LP4 $(0.5,1.5 \mu \mathrm{M})$ and then with tetramethylrhodamine methyl ester (TMRM) to analyze $\triangle \Psi \mathrm{m}$ in the absence of massive cell death, with TMRM fluorescence being measured from gated living cells alone (Figure 2A). Antp-LP4 treatment of CLL-derived PBMCs resulted in reduced TMRM fluorescence (Figures $2 \mathrm{Bc}$ and $2 \mathrm{Bd}$ ) while carbonyl cyanide-p-trifluoromethoxyphenylhydrazone (FCCP)treated cells served as a control for depolarization (Figures 2Bb). Quantitation of the results further indicates $\triangle \Psi \mathrm{m}$ dissipation following Antp-LP4 treatment of CLL cells (Figure 2C).

The Antp-LP4-mediated decrease in $\triangle \Psi \mathrm{m}$ was expected to decrease cellular ATP levels. PBMCs were incubated for a short time $(5 \mathrm{~min})$ with low peptide concentrations $(0.5$, $1.5 \mu \mathrm{M})$ to rule out the possibility that any decrease in ATP levels is not due to cell death (Figure 2D). PBMCs from CLL patients exhibited a $50 \%$ decrease in cellular ATP levels following such treatment (1.5 $\mu \mathrm{M}$ peptide) (Figure 2D). These results clearly indicate that in response to Antp-LP4 treatment, cell energy production is decreased.

Antp-LP4 induces detachment of mitochondrial-bound HK and VDAC1 oligomerization. HK is over-expressed in highly energy-demanding cancer cells. ${ }^{8}$ In CLL, isoform 1 (HK-I) but not 2 (HK-II) is slightly over-expressed (by $30 \%$ ) at the protein level. ${ }^{23}$ Antp-LP4 interfered with the interactions of $\mathrm{HK},{ }^{12,16,17} \mathrm{Bcl}-2,{ }^{15}$ and $\mathrm{Bcl}-\mathrm{xL}^{14}$ with VDAC1 and abolished their anti-apoptotic effects.

Owing to the limited access to CLL samples and their low stability, we used MEC-1 cells to analyze peptide-induced displacement of mitochondrial-bound HK. immunocytochemistry of untreated MEC-1 cells, using anti-HK-I antibodies, showed punctuated fluorescence (Figure 3Aa), indicating mitochondrial distribution. By contrast, cells incubated with Antp-LP4 showed weak and diffuse 

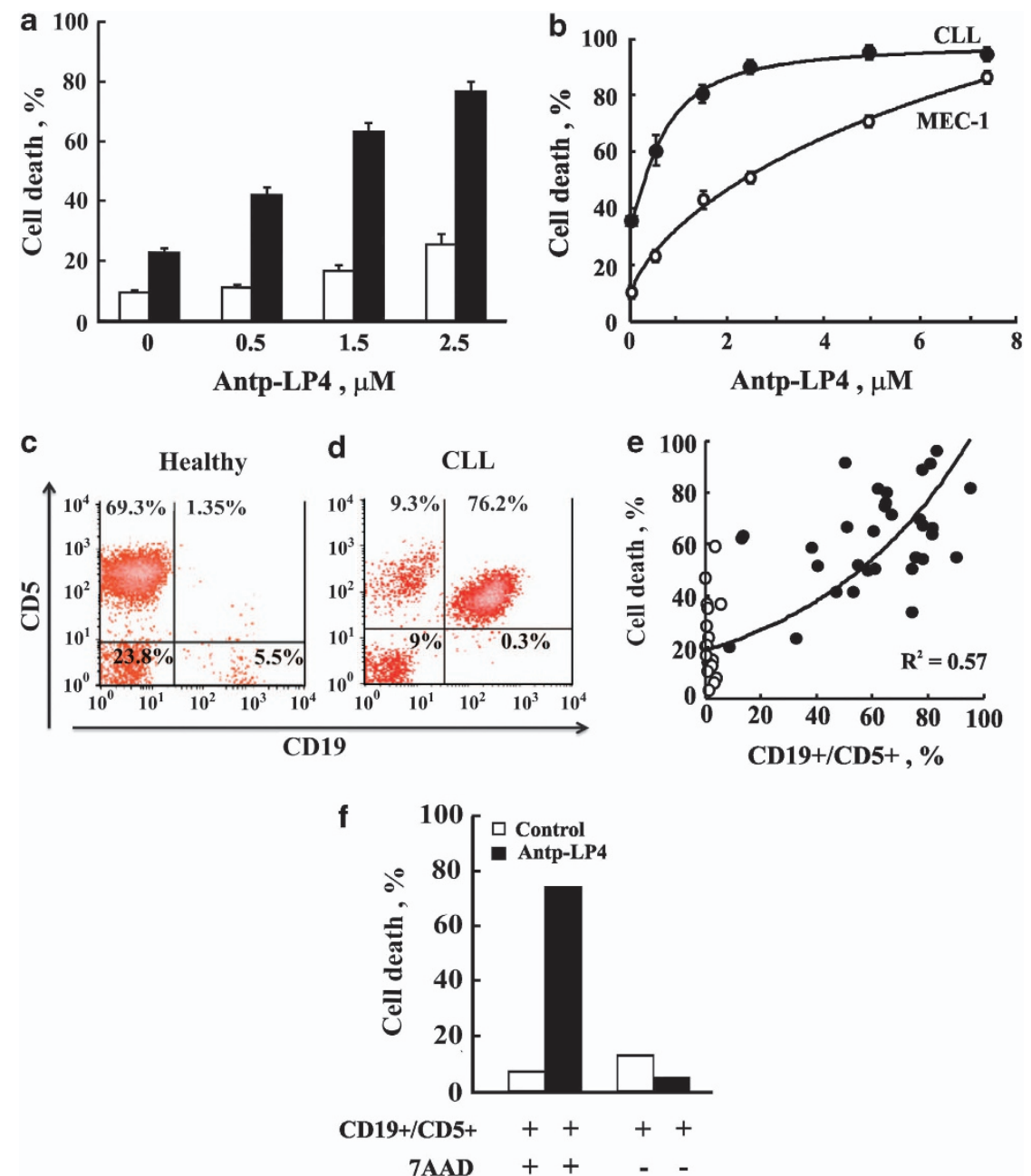

Figure 1 Antp-LP4 peptide specifically induces CLL cancer cell death. PBMCs isolated from CLL patients and healthy individuals were incubated for 90 min with Antp-LP4. (a) Quantitation FACS analysis of Antp-LP4-induced death of PBMCs from CLL donors $(n=51)$ and healthy patients $(n=34)$. (b) A CLL patient-derived PBMCs ( $)$ and MEC-1 cells $(O)$ were incubated with the peptide and analyzed for cell death. Values represent mean $\pm S E(n=6)$. (c-f) The percentage of CD19+/CD5 $+(C L L$ cancerous cells) expressing cells in a CLL patient's PBMCs (d) and in a healthy donor (c) was determined using monoclonal antibodies directed to CD19 and CD5, and FACS analysis. (e) The percentage of cell death induced by Antp-LP4 $(1.5 \mu \mathrm{M})$ is plotted as function of the percentage of CD19+/CD5 + cells for each patient $(\mathbf{O})$ or healthy donor $(O)$. (f) CLL cells were incubated for $90 \mathrm{~min}$ with Antp-LP4 $(2.5 \mu \mathrm{M})$ and analyzed by FACS for cells expressing CD19+ and CD5 + and undergoing cell death using 7AAD (instead of PI)

Table 1 Selected VDAC1-based peptides induce cell death in PBMCs from CLL patients and in MEC-1 cells

\begin{tabular}{|c|c|c|c|c|c|}
\hline \multirow[b]{2}{*}{ No } & \multirow[b]{2}{*}{ Peptide } & \multirow[b]{2}{*}{ Peptide sequence } & \multirow[b]{2}{*}{ AA } & \multicolumn{2}{|c|}{$\mathrm{IC}_{50} \mu \mathrm{M}$} \\
\hline & & & & CLL & MEC-1 \\
\hline 1 & Antp-LP4 & RQIKIWFQNRRMKWKK-LP4 & 43 & $0.7 \pm 0.1(n=10)$ & $2.5 \pm 0.1(n=16)$ \\
\hline 2 & D-Antp-LP4 & RQIKIWFQNRRMKWKK-LP4 & 43 & $0.7 \pm 0.4(n=3)$ & $1.6 \pm 0.3(n=5)$ \\
\hline 3 & Min-Antp-LP4 & KRRMKWKK-LP4 & 33 & $0.3 \pm 0.1(n=3)$ & $1.7 \pm 0.4(n=7)$ \\
\hline 4 & D-MinAntp-LP4 & KRRMKWKK-LP4 & 33 & $0.6 \pm 0.2(n=3)$ & $1.4 \pm 0.1(n=5)$ \\
\hline 5 & Tf-LP4 & HAIYPRH-LP4 & 34 & $1.7 \pm 0.2(n=4)$ & $3.6 \pm 0.2(n=18)$ \\
\hline 6 & Tf-D-LP4 & HAIYPRH-LP4 & 34 & $1.2 \pm 0.1(n=3)$ & $1.8 \pm 0.2(n=3)$ \\
\hline 7 & N-Ter-Antp & N-Ter-RQIKIWFQNRRMKWKK & 42 & $3.2 \pm 0.5(n=6)$ & $4.2 \pm 0.2(n=12)$ \\
\hline 8 & D-N-Ter-Antp & N-Ter-RQIKIWFQNRRMKWKK & 42 & $1.5 \pm 0.6(n=3)$ & $2.2 \pm 1.6(n=3)$ \\
\hline 9 & $\Delta(21-26)-\mathrm{N}-T e r-A n t p$ & $\Delta(21-26)-N-T e r-R Q I K I W F Q N R R M K W K K$ & 36 & $>12.5(n=3)$ & $>12.5(n=3)$ \\
\hline 10 & $\Delta(1-14)-\mathrm{N}-$ Ter-Antp & $\Delta(1-14)-N-T e r-R Q I K I W F Q N R R M K W K K$ & 28 & $2.2 \pm 0.2(n=3)$ & $3.3 \pm 0.2(n=7)$ \\
\hline 11 & D- $\Delta(1-14)-N-T e r-A n t p$ & $\Delta(1-14)-N-T e r-R Q I K I W F Q N R R M K W K K$ & 28 & $1.3 \pm 0.1(n=2)$ & $2.1 \pm 0.3(n=3)$ \\
\hline
\end{tabular}

Cells were incubated for 90 min with increasing concentrations of the indicated peptide and death was analyzed by PI staining and FACS. The peptide concentration yielding half-maximal cell death $\left(\mathrm{IC}_{50}\right)$ is indicated. AA indicates the number of amino acids comprising the peptide. Data represent mean $\pm \mathrm{S}$. $\mathrm{E}$., while $n$ is the number of repeats performed.

LP4: SWTWEKKLETAVNLAWTAGNSNKWTWK

N-Ter: MAVPPTYADLGKSARDVFTKGYGFGL. 

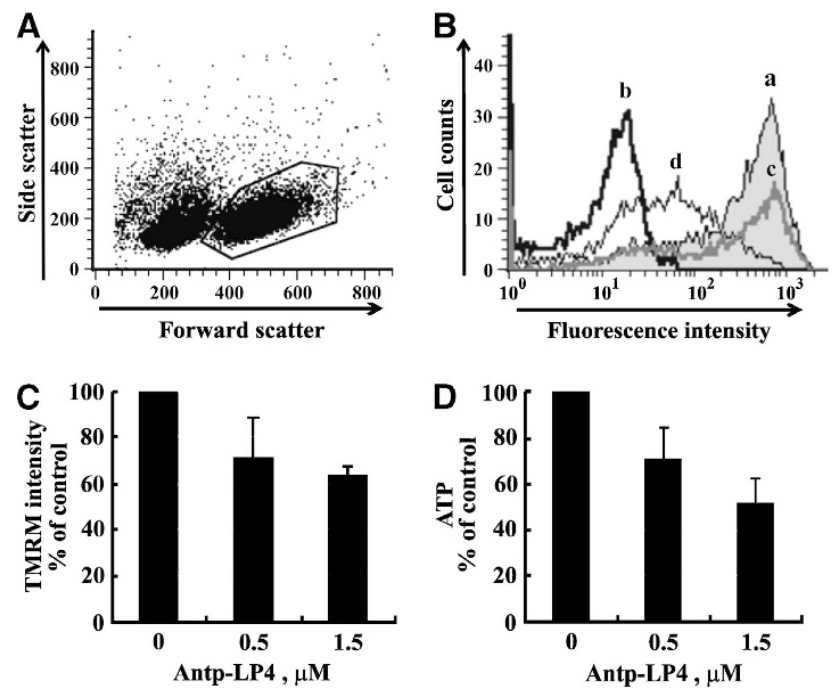

Figure 2 Antp-LP4 induces mitochondrial inner membrane depolarization and reduced cellular ATP levels. CLL cells were incubated for 15 min with 0.5 or $1.5 \mu \mathrm{M}$ Antp-LP4 and analyzed for mitochondrial $\Delta \Psi$ with TMRM. (A) Representative FACS analysis of TMRM fluorescence of living cells (gated as shown). (B) Quantitation of TMRM fluorescence intensity (geometric mean) of (a) nontreated (black line), (b) FCCP-treated $(10 \mu \mathrm{M})$ (control for depolarization) and 0.5 (c) or $1.5 \mu \mathrm{M}$ (d) Antp-LP4-treated cells. (C) TMRM fluorescence intensity as a percentage of control $(n=3)$. (D) ATP levels in CLL patient-derived PBMCs treated for $5 \mathrm{~min}$ with or without the indicated concentration of Antp-LP4. In each treatment, cells were also stained with trypan blue to determine cell viability

fluorescence, reflecting displacement of HK from the mitochondria (Figure $3 \mathrm{Bb}$ ). The decrease in the total fluorescence intensity may reflect $\mathrm{HK}$ degradation upon its detachment. This is further supported by western blotting of the cytosolic and mitochondrial fractions of cells untreated or treated with the peptide, where decreased HK levels were noted in those fractions generated from the peptide-treated cells (data not shown).

VDAC1 oligomerization was demonstrated to be coupled to apoptosis induction. ${ }^{24,25}$ Antp-LP4-treated MEC-1 cells were subjected to chemical cross-linking with the membranepermeable cross-linker, ethylene glycol-bis(succinimidyl succinate) (EGS). VDAC1 oligomerization was then assessed by SDS-PAGE and immunoblot, using anti-VDAC1 antibodies (Figure $3 \mathrm{C}$ ). The formation of VDAC1 dimers and higher molecular mass complexes was enhanced over twofold upon exposure to Antp-LP4 (Figures $3 \mathrm{C}$ and D), with the concentration of EGS required to attain $50 \%$ of the maximal level of dimers being decreased twofold.

CLL patients express high levels of VDAC1 as compared with healthy donors (Figure 3E). Importantly, the extent of cell death induced by the peptide is positively correlated with VDAC1 levels (Figure 3F, $R^{2}=0.55$ ).

Antp-LP4 induces Cyto $c$ release and apoptotic cell death. Next, the activity of the peptide in inducing Cyto $c$ release and subsequent apoptosis was analyzed in MEC-1 cells by immunocytochemistry using anti-Cyto $c$ antibodies. Representative confocal images of control cells show that the fluorescence is punctuated, suggesting mitochondrial distribution of Cyto $c$ (Figure 4A). The staining, however, became diffuse and weaker upon exposure to the peptide (Figure 4B), reflecting Cyto $c$ release from mitochondria to the cytosol and probably its degradation. This is further demonstrated by western blot analysis of the cytosolic fraction of cells untreated or treated with the peptide (Figure 4C). In cells treated with low concentration of the peptide, Cyto $c$ was detected in the cytosolic fraction, whereas at higher peptide concentration, Cyto $c$ could not be detected (Figure 4C), and its total amount in the sample before separation of the cytosolic fraction was decreased (data not shown), suggesting its degradation. Cyto $c$ degradation in the cytosol upon apoptotic induction and mediated via ubiquitination has been demonstrated. ${ }^{26}$

The confocal images also show that upon peptide treatment, the cell nucleus undergoes the characteristic morphological changes associated with apoptosis, such as clumping of nuclear chromatin and condensation of nuclei (Figure 4B).

To further demonstrate the induction of apoptosis by the peptide, we used fluorescence and electron microscopy (EM) to demonstrate apoptosis-associated morphological changes. Antp-LP4 treatment of MEC-1 cells stained with acridine orange/ethidium bromide ${ }^{12}$ showed membrane blebbing and chromatin condensation, the hallmark of apoptosis (Figure 4D). With increased peptide concentration, increased numbers of dark orange-stained cells were observed, reflecting cells in a late apoptotic stage. Quantitation of these results shows that the peptide induced apoptotic cell death in $>95 \%$ of the cells (Figure 4E).

Antp-LP4-induced apoptosis was also revealed by following Annexin V-FITC-labeling of apoptosis-induced phosphatidylserine (PS) exposure (early apoptotic state), and PI staining of nuclear (late apoptotic state) (Figure 4F).

Morphological changes in the nuclear chromatin of AntpLP4-treated cells were also detected by EM. Representative micrographs show that in both control and peptide-treated cells, the outer plasma membrane is visibly intact (Figures 5A and B). However, peptide-treated cells show the typical morphologic changes associated with apoptosis (Figure 5B). These changes include condensation of nuclei (Figure $5 \mathrm{Ba}$ ), DNA fragmentation (Figures $5 \mathrm{Bb}$ and $\mathrm{c}$ ) and possible apoptotic body formation (Figure 5Bb).

Other cell-penetrating VDAC1-based peptides induce death of CLL cells. Twenty-seven versions of cell-penetrating VDAC1-based peptides were designed with the aim of preventing degradation by peptidases and tested for their cell death-inducing ability toward CLL and MEC-1 cells, as reflected in $I_{50}$ values (Figure 6, Table 1 and Supplementary Table 2). The VDAC1-based sequences and that of the fused cellpenetrating peptide of three initial peptides tested are presented (Figure 6a).

The cell-penetrating Antp sequence of Antp-LP4 was replaced with a human transferrin receptor ( $\mathrm{hTfR}$ )-recognition sequence, HAIYPRH $(\mathrm{Tf})^{27}$ to form Tf-LP4. hTfR is overexpressed in many cancers, ${ }^{27}$ including $\mathrm{CLL}^{28}$ (Figures $6 \mathrm{~b}$ and $\mathrm{c}$ ). Tf-LP4 induced cell death in CLL patient-derived PBMCs and in MEC-1 cells (Table 1). The TAT-LP4, bearing the HIV-1 Tat-dependent transactivation peptide TAT, shown to confer cell permeability, ${ }^{29}$ was less effective in inducing cell 
A

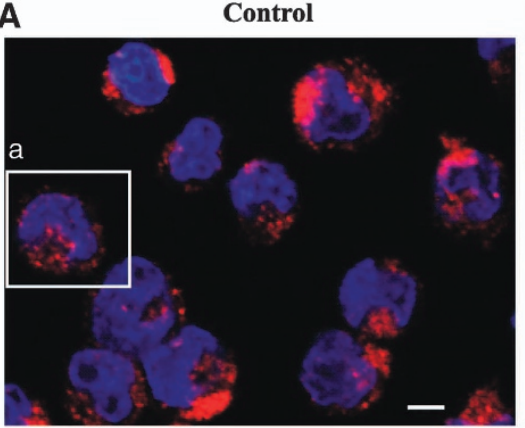

B
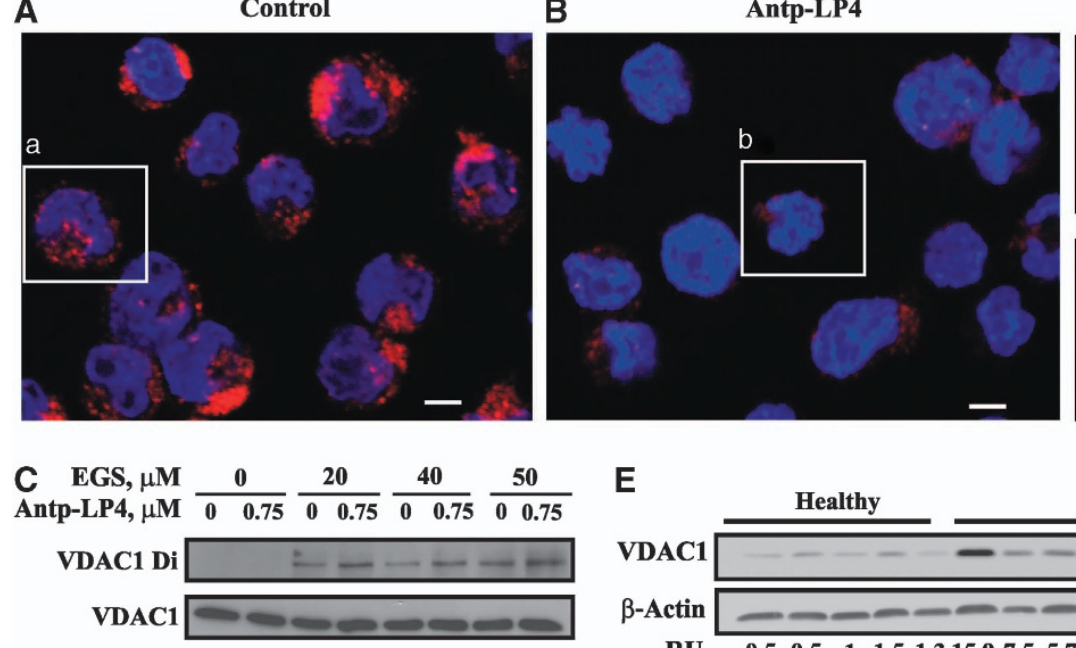

D

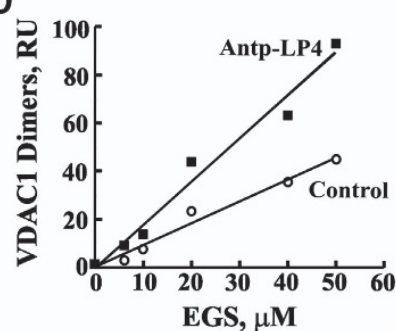

E
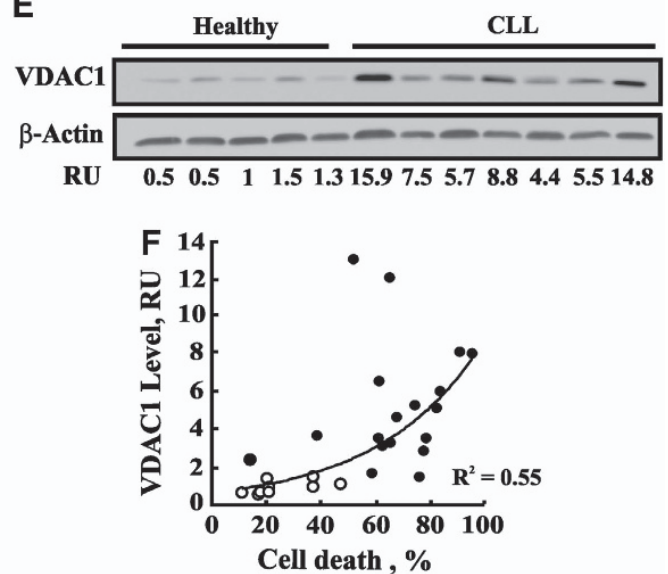

Figure 3 Antp-LP4 induces HK displacement and VDAC1oligomerization. MEC-1 cells were incubated without (control, A) or with Antp-LP4 (0.5 $\mu$ M, 90 min, B) and HK detachment from the mitochondria was analyzed by immunostaining using anti-HK-I antibodies. Representative microscopic fields from one of three similar experiments are shown. (a) and (b) show an enlargement of a representative cell from A and B, respectively. Scale bar, $5 \mu \mathrm{m}$. To assess VDAC1 oligomerization, control and Antp-LP4-treated $(0.75 \mu \mathrm{M}, 40 \mathrm{~min}) \mathrm{MEC}-1$ cells were incubated with the indicated concentration of EGS ( $\left.15 \mathrm{~min}, 30^{\circ} \mathrm{C}\right)$. Cross-linking was terminated by sample buffer addition and heating $\left(70^{\circ} \mathrm{C}, 10 \mathrm{~min}\right)$. Cells were subjected to SDS-PAGE, followed by immunoblotting using anti-VDAC1 antibodies and HRP-conjugated secondary antibody, visualized by enhanced chemiluminescence (C). Quantitation of immuno-stained VDAC1dimers as a function of EGS concentration (D). VDAC1 over-expression in CLL patients-derived PBMCs and healthy donors as probed with anti-VDAC1and anti- $\beta$-actin antibodies. A representative immunoblot and its quantitation (RU) are shown (E). VDAC1 levels in PBMCs isolated from healthy donors $(n=9)(0)$ or CLL patients $(n=18)(0)$ were plotted as a function of the percentage of cell death induced by Antp-LP4 (1.5 $\mu$ M) for each individual $\left(R^{2}=0.55\right)(\mathbf{F})$

death (Supplementary Table 2) of both CLL PBMCs and MEC-1 cells. This peptide thus offers no advantage over Antp-LP4 or Tf-LP4.

The D-enantiomers of the peptides were also tested, with all-D amino-acid Antp-LP4 and N-Ter-Antp peptides being as, if not more effective, than the corresponding L-version in inducing cell death of CLL PBMCs and MEC-1 cells (Figures $6 \mathrm{~d}-\mathrm{f}$ and Table 1). D-Tf-LP4 was less active, while when only the LP4 portion was in the D-version (Tf-D-LP4) the peptide was as effective as was the all L-version (Figure $6 \mathrm{~d}$, Table 1 and Supplementary Table 2).

Shortening the Antp moiety to a minimal sequence conferring cell permeability ${ }^{30}$ resulted in a very active peptide (Min-Antp-LP4), with the D-Min-Antp-LP4 being active as D-Antp-LP4 (Figure 6e). Min-Antp-LP4 is eight residues shorter and twofold more effective $\left(\mathrm{IC}_{50}\right.$ of $0.3 \mu \mathrm{M}$ versus $0.7 \mu \mathrm{M}$ for Antp-LP4) (Table 1).

Other versions of Antp-LP4 and Tf-LP4, including linearized peptides lacking the tryptophan zipper, cyclic peptides formed upon introducing cysteines to form thiol bonds, and peptides lacking the cytosol-facing sequence found in LP4 (no loop), were all less active than were the parent peptides. Finally, blocking the Tf-LP4 peptide N-terminus by acetylation or the C-terminus by amidation decreased peptide efficacy up to fourfold, relative to Tf-LP4 (Supplementary Table 2).

The cell killing activity of another VDAC1-derived peptide, the (1-26)-N-Ter-Antp peptide (Figure 6a), ${ }^{13-15,17}$ was also tested and found to induce cell death when Antp was C-terminally fused (Figure $6 f$ and Table 1). A peptide bearing an N-terminally fused Antp moiety was much less effective (data not shown). Fusing N-Ter-Antp with Tf yielded a lessactive peptide (Supplementary Table 2). The D-enantiomer of $\mathrm{N}$-Ter-Antp was twofold more active than was the L-peptide (Table 1 and Figure 6f). Shortening the (1-26)-N-Ter-Antp peptide by up to 14 residues $(\triangle(1-14) N$-Ter-Antp) did not significantly modify the activity, whereas deleting six residues from the $\mathrm{C}$-terminus of the peptide, including the GXXXG motif, yielded a non-active peptide (Table 1 and Supplementary Table 2). Combining Antp-LP4 with N-Ter-Antp produced an additive effect (data not shown), suggesting that binding of a single peptide to the anti-apoptotic protein is sufficient to interfere with its binding to VDAC1. 

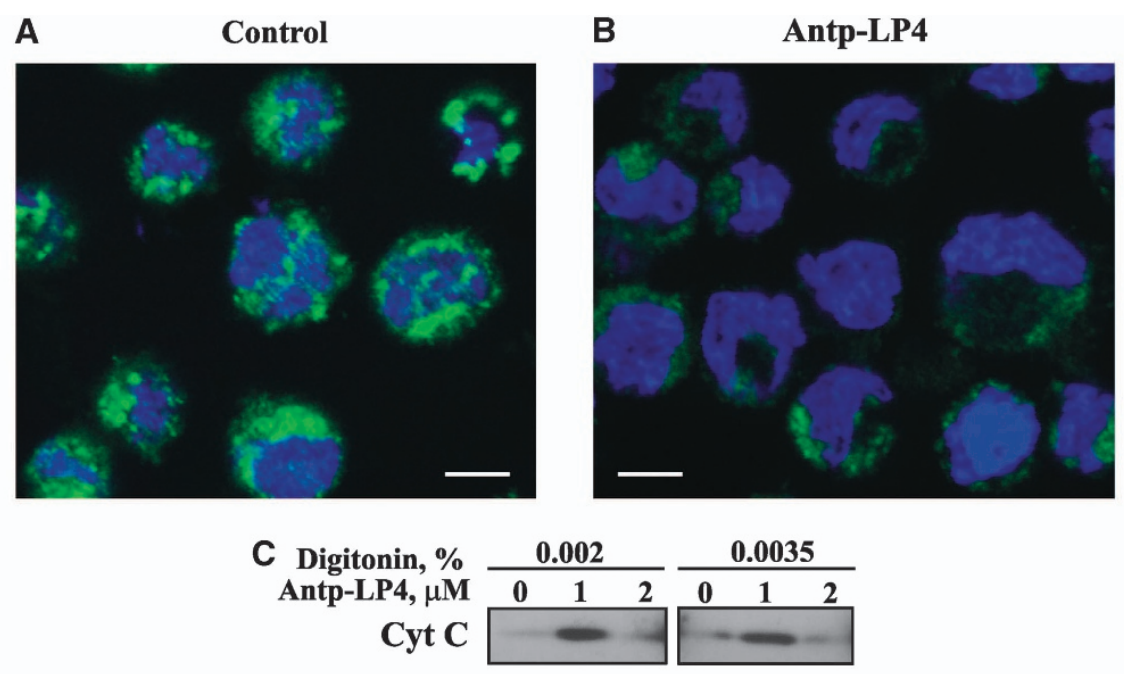

D Antp-LP4, $\mu$ M 0
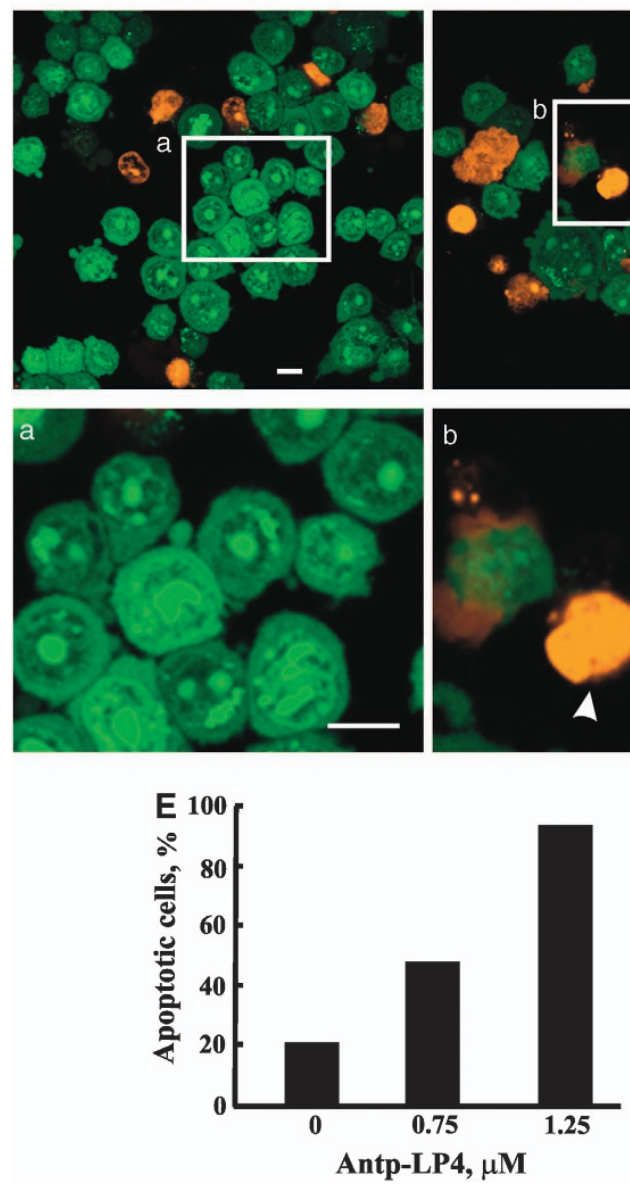

0.75
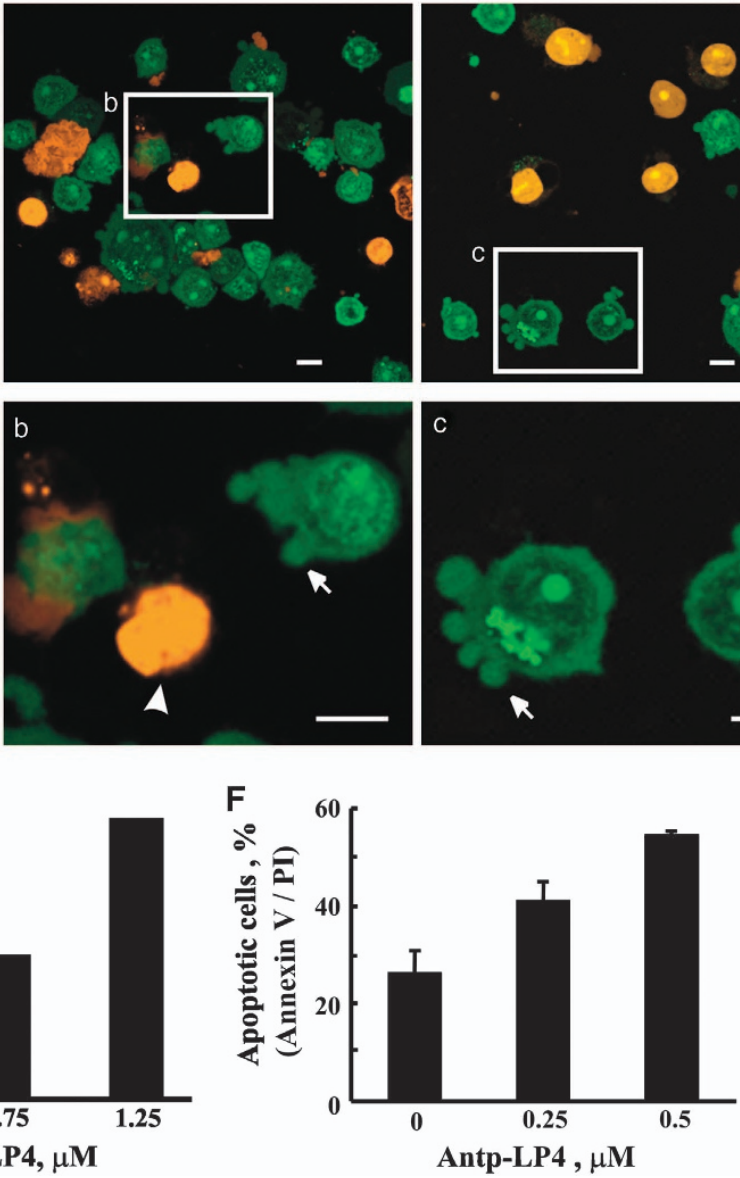

Figure 4 Antp-LP4 induces cytochrome $c$ release and apoptotic cell death. MEC-1 cells were incubated without (A) (control), or with Antp-LP4 (B) (1.5 $\mu$ M, 90 min), and cytochrome $c$ release was analyzed using anti-cytochrome $c$-specific antibodies. Scale bars, $5 \mu \mathrm{m}$. (C) MEC-1 cells were incubated with and without the indicated concentration of Antp-LP4, treated with digitonin $(0.002 \%$ or 0.0035$)$ and release of Cyto $c$ from mitochondria to the cytosolic fraction was analyzed by immunoblotting, as described in Materials and Methods. (D) CLL patient-derived PBMCs were treated with Antp-LP4 for 15 min and then stained with acridine orange/ethidium bromide. Squares in (D) point to the area enlarged in a-C. The arrow and arrowhead indicate cells in early and late apoptotic states with membrane blebbing (a hallmark of apoptosis), respectively. Scale bar, $10 \mu \mathrm{m}$. (E) Quantification of apoptosis in control cells and cells treated with the indicated Antp-LP4 concentrations for 40 min. (F) CLL patients-derived PBMCs were incubated for short time (15 min) with low concentrations of Antp-LP4 $(0.25$ and $0.5 \mu \mathrm{M})$ and stained with FITC-Annexin V followed by PI staining. Apoptotic cell amounts were analyzed with an ImageSteam multi-spectral imaging flow cytometer after debris was eliminated 
A

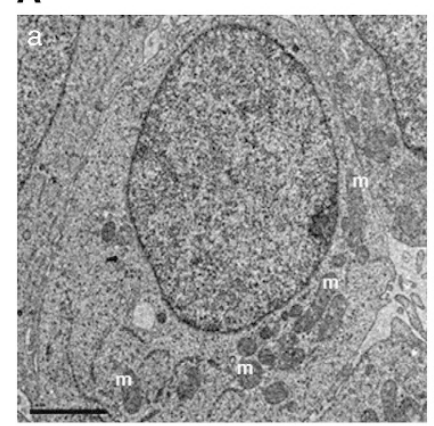

B

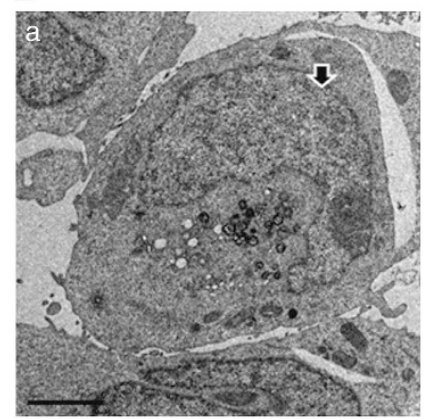

Control

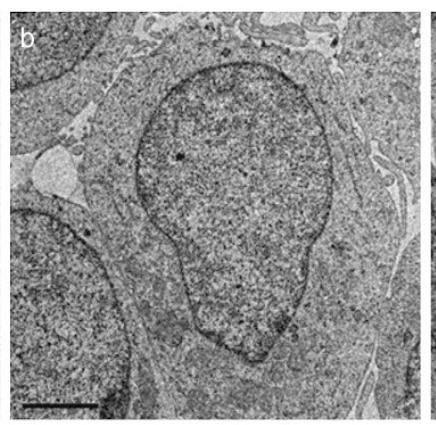

Antp-LP4

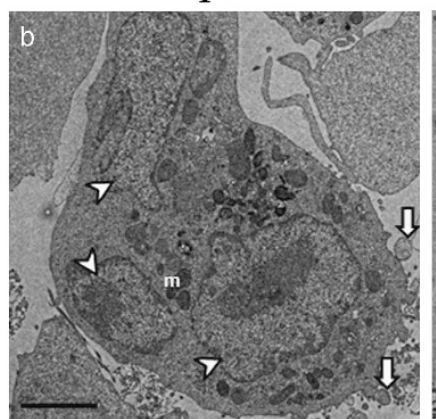

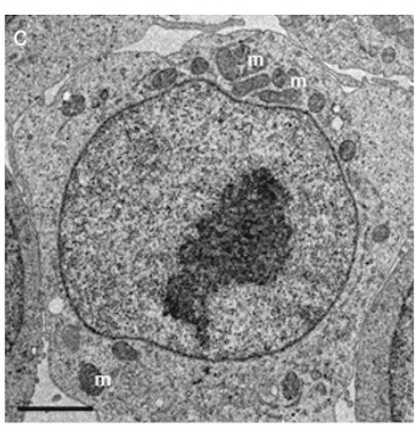

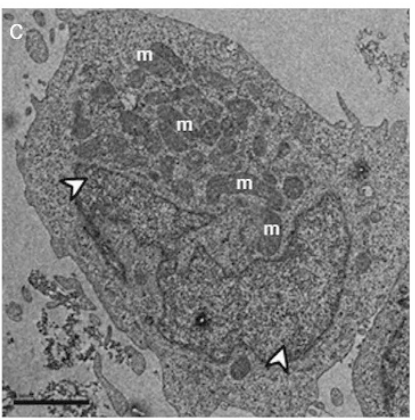

Figure 5 Electron microscopy visualization of Antp-LP4 induces apoptotic cell death MEC-1 cells left untreated (A) (control) or treated with $1.5 \mu \mathrm{M}$ of Antp-LP4 (B) were examined by EM. Cells from three representative control and peptide-treated samples are presented. In the peptide-treated cells, typical morphologic changes associated with apoptosis, such as condensation of nuclei ( $\mathrm{Ba}$, black arrow), DNA fragmentation ( $\mathrm{Bb}, \mathrm{Bc}$, white arrowheads) and membrane blebbeing ( $\mathrm{Bb}$, white arrow), were noted. Mitochondrion is indicated by letter $\mathrm{m}$

\section{Discussion}

Antp-LP4 and its derivatives selectively kill PBMCs from CLL patients. We selected CLL as a model to elucidate the effectiveness of VDAC1-based peptides as anti-cancer therapy, as the failure of $B$ lymphocytes to undergo apoptosis is the main cause of this disease. ${ }^{2}$ In a series of ex vivo experiments, we demonstrated that Antp-LP4 effectively induced cell death in PBMCs derived from 51 CLL patients but only slightly affected cells from 34 healthy donors (Figure 1a). In addition, a clear correlation between the degree of cell death induced by the peptide and relative cancer cell levels (expressing CD5 $+/ C D 19+$ ) in each patient was obtained (Figure 1e). Furthermore, the results show that CD5 + /CD19 + cells represent the peptide-sensitive cells in the population (Figure 1f). Together, these findings indicate the high specificity of Antp-LP4 toward cancer cells and provide new opportunities for the development of novel and effective therapies for this presently incurable disease.

To optimize Antp-LP4 and N-Ter-Antp peptides with respect to stability, length and specific targeting to cancer cells, 27 versions of these peptides (Supplementary Table 2 and Table 1) were designed and tested for their efficacy in inducing the death of CLL-derived lymphocytes and MEC-1 cells. The modifications introduced into Antp-LP4 and N-TerAntp included using a shorter sequence, and/or D-amino acids, leading to effective and more stable peptides. Some VDAC1-based peptides showed diminished activity. Deleting ${ }^{1-14}$ residues in the 1-26-N-terminal peptide improved peptide efficacy, while deleting six C-terminal residues ${ }^{21-26}$ strongly decreased the activity (Table 1). Interestingly, the deleted sequence represents the GXXXG motif implicated in protein dimerization ${ }^{31}$ and is found in $\mathrm{Bcl}-\mathrm{xL},{ }^{32} \mathrm{Bak}$ and $\mathrm{Bcl} 2$.

The Tf-LP4 peptide, comprising a TfR targeting peptide, HAIYPRH, ${ }^{33}$ fused to LP4, induced death twofold less effectively than did Antp-LP4 (Table 1). However, Tf-LP4 allows specific targeting to CLL cells over-expressing hTfR (Figures $6 b$ and $c$ ). Tf fused to peptides, siRNA or small molecules directed internalization into cells expressing $\mathrm{hTfR}^{33-36}$

The results of this comprehensive screen led to the identification of four optimized peptides, Tf-D-LP4, D-AntpLP4, D-MinAntp-LP4 and D- $\triangle(1-14)-N-T e r-A n t p$ (highlighted in gray in Table 1), that are expected to be protease-resistant and will be further studied in vivo using a human CLL mouse model.

The mode of action of Antp-LP4. Our results indicate that the VDAC1-based peptides have a double action, affecting both cell energy production and inducing apoptosis (Figure 7).

Altered energy metabolism, including enhanced aerobic glycolysis, is a fundamental phenotype of malignant tumors. ${ }^{7,37,38}$ Mitochondrial-bound HK is markedly elevated in highly glycolytic cancer cells, ${ }^{8}$ supporting aerobic glycolysis, ${ }^{39}$ critical for the stability of mitochondria ${ }^{40}$ and confers resistance to apoptosis. ${ }^{10,12,13,16,17,40,41} \mathrm{HK}$ was reported not to be over-expressed in CLL. ${ }^{42}$ We confirmed this for HK-II but 

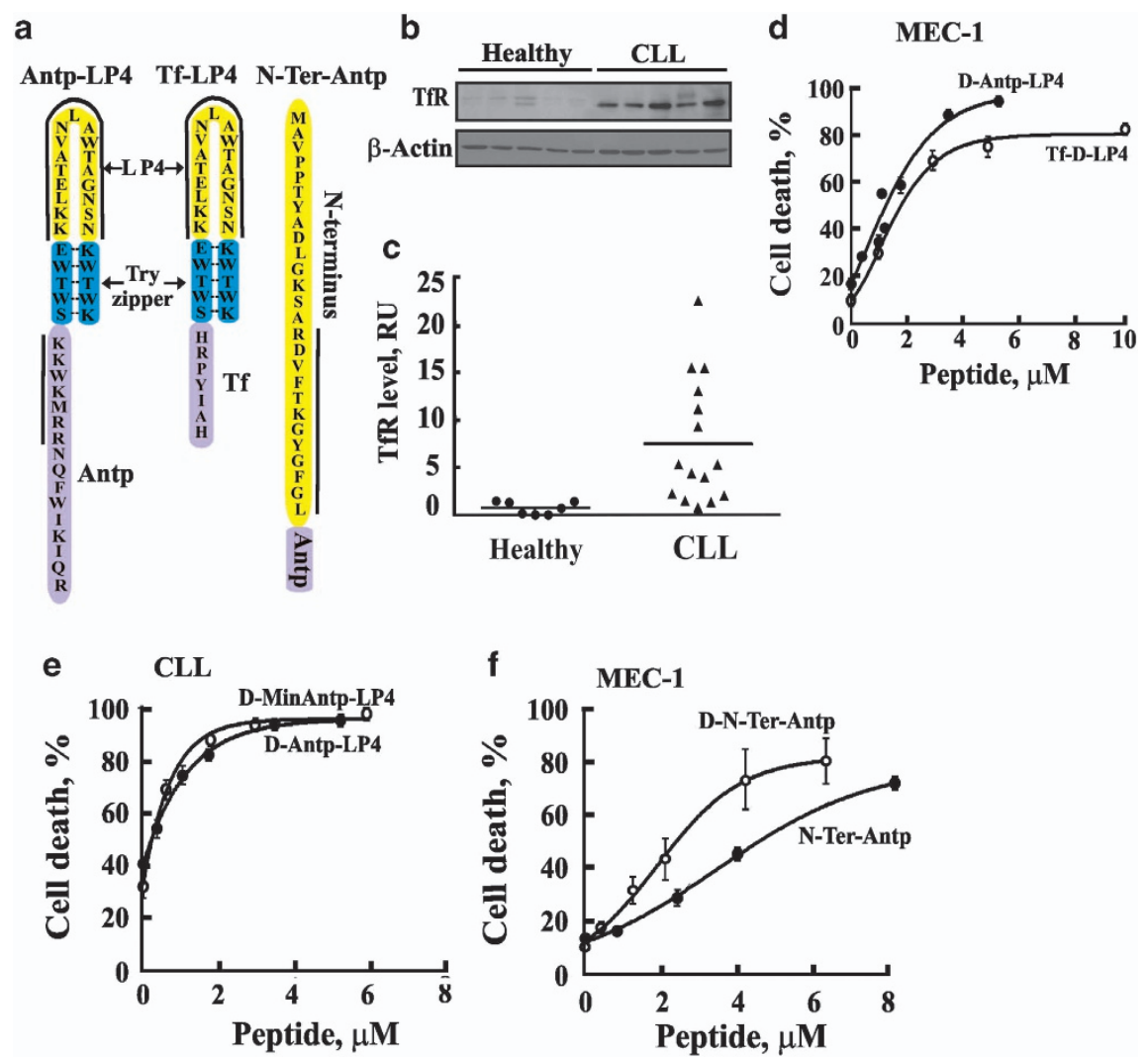

Figure 6 Induction of cell death by various VDAC1-based peptide derivatives. (a) Schematic illustration of the structures of the major VDAC1-based peptides studied. Loop-shaped Antp-LP4, Tf-LP4 and N-Ter-Antp VDAC1-based peptides are shown. VDAC1-derived sequences are in yellow, the cell-penetrating peptide (Antp or Tf) is in purple and the loop shape stabilized by a tryptophan zipper is in blue. The solid black lines mark the most important amino acids for the activity of a peptide. (b) TfR levels in PBMCs from CLL patients and healthy donors as analyzed by immunoblot using anti-TfR antibodies. (c) Scatter plots of TfR expression level for each subject (controls ( $\bullet$ and CLL patients $(\boldsymbol{\Delta})$ ) is presented. Statistics were calculated with GraphPad Prism. Vertical bars indicate mean value, median. (d-f) VDAC1-based peptides induce cell death in CLL patient-derived PBMCs (d) or MEC-1 cells (e and f). Cells were incubated for 90 min with the indicated peptide and assayed for cell death using PI staining and FACS analysis

found HK-I over-expression in some CLL patients (by 30\%) at the protein level and no change at the mRNA level. ${ }^{23}$ We have previously demonstrated that Antp-LP4 detached HK from mitochondrial VDAC1 in T-Rex-293 cells, and from rat brain and BCL1 leukemia tumors. ${ }^{17}$ Although the same occurred in MEC-1 cells, we found that upon its displacement, HK undergoes degradation. Such Antp-LP4-induced HK displacement affects the overall cellular bioenergetics as reflected in the peptide-induced decrease in $\triangle \Psi \mathrm{m}$ and cellular ATP levels (Figure 2).

The proposed mechanism (Figure 7) whereby VDAC1based peptides induce apoptosis is related to the overexpression of VDAC1 in CLL, demonstrated here for the first time (Figure 3E), with correlation seen between VDAC1 levels and cell death induced by Antp-LP4 (Figure 3F). VDAC1 overexpression might offer the following advantages to cancer cells that can be blocked in the presence of the peptides: (i) over-expressed VDAC1 presents anchoring sites for HK, allowing direct excess to mitochondrial ATP and an increased glycolytic rate. ${ }^{10}$ This coupling is impaired by the peptide detaching HK, leading to decreased glycolysis, energy and precursor production and allows apoptosis induction. ${ }^{13,17,43}$ (ii) Over-expressed VDAC1 offers binding sites for $\mathrm{Bcl} 2$ and $\mathrm{Bcl}-\mathrm{xL}$, allowing these proteins to mediate their anti-apoptotic effects. ${ }^{14,15}$ However, when the peptide interferes with their association with VDAC1, apoptosis ensues. ${ }^{14,15}$ Indeed, cells treated with the peptide show characteristic features of apoptosis (Figures 3-5).

We have shown that VDAC1 oligomerization is coupled to apoptosis induction. ${ }^{24}$ VDAC1 over-expression encourages its oligomerization, leading to apoptosis in the absence of any apoptosis inducer. ${ }^{13}$ We propose that displacement of HK, $\mathrm{Bcl}-\mathrm{xL}$ and $\mathrm{Bcl}-2$ from VDAC1 encourages VDAC1 oligomerization (Figure 3), due to the high concentration of free VDAC1 molecules, leading to apoptosis. Indeed, Antp-LP4 induced release of Cyto $c$, and other hallmarks of apoptosis, as reviled by both confocal and EM, including membrane blebbing, PS surface exposure, and nuclear condensation and fragmentation (Figures 3-5). Thus, VDAC1-based peptides mode of action perturbed cell energy metabolism and inducing apoptosis.

The advantages of using VDAC1-based peptides as a potential anti-cancer agent in CLL. Presently, treatment of CLL involves steroids, alkylation agents, purine analogs (particularly fludarabine), combination chemotherapy ${ }^{44}$ and monoclonal antibodies. ${ }^{45}$ However, most available therapeutic options are associated with significant toxicity, sustained 


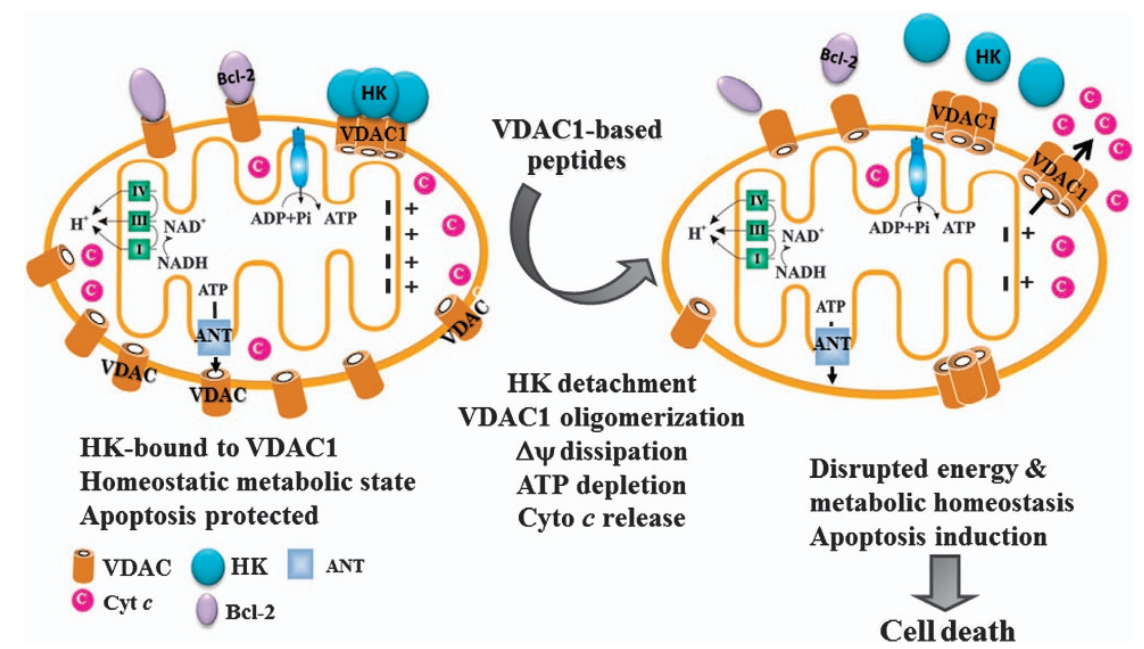

Figure 7 Proposed mode of action of Antp-LP4 leading to mitochondria-mediated cell death. In the CLL B-lymphocyte mitochondrion (left), VDAC1 is over-expressed and associated with $\mathrm{Bcl}-2$ and $\mathrm{HK}, \Delta \Psi \triangle$ is maintained and apoptosis cannot be activated. The cell thus remains in homeostasis with respect to energy production and is protected from apoptosis. Following Antp-LP4 peptide treatment (right), anti-apoptotic HK and Bcl-2 interact with the peptide and disassociate from VDAC1. These changes lead to $\triangle \Psi$ dissipation, decreased ATP production, mitochondrion dysfunction, VDAC1 oligomerization and Cyto $c$ release, events ultimately leading to cell death

immunosuppression and drug resistance. ${ }^{46,47}$ In this respect, we found that fludarabine at relatively high concentrations had only a weak cell killing effect on PBMCs isolated from CLL patients (data not shown).

Interestingly, oblimersen (G3139), an anti-sense oligonucleotide being studied as a possible treatment for several types of cancer, including CLL, ${ }^{48}$ is proposed to act via VDAC1. ${ }^{49}$ Although CLL cells are sensitive to ABT-737induced apoptosis, they develop resistance within $24 \mathrm{~h}$ due to the increased expression of BCL2A $1,{ }^{50} \mathrm{Bcl}-x \mathrm{~L}$ or Bcl-w. ${ }^{51}$

Therapeutic cell-penetrating peptides have generated interest as potential anti-cancer agents, owing to their specificity, rapid clearance from systemic circulation, ability to penetrate tumor tissue and relatively low cost of synthesis. ${ }^{52}$ However, their use in vivo is limited by low stability and poor tumor penetration. Still, several peptides gained first marketing approval for clinical use in 2012, among them is carfilzomib for the treatment of multiple myeloma. Today, many other candidates are in the pipeline, including 74 in Phase I/II or Phase II studies. ${ }^{53}$

To conclude, here, we demonstrated the high effectiveness of cell-penetrating VDAC1-based peptides with perceived specificity toward cancerous cells. The dramatic cancer cell death induction by VDAC1-based peptides results from their multiple effects, including impairment of energy homeostasis and overcoming the protective and pro-survival actions taken by cancer cells. As such, these VDAC1-based peptides could have a wide therapeutic index and represent good drug candidates for treating CLL.

\section{Materials and Methods}

Materials. 7-Amino-actinomycin, FCCP, leupeptine, phenylmethylsulfonyl fluoride (PMSF), PI, TMRM, DAPI, 4', 6-diamidino-2-phenylindole and trypan blue were purchased from Sigma (St. Louis, MO, USA). Annexin-V was obtained from Alexis Biochemicals (Lausen, Switzerland). A CellTiter-Glo Luminescent Cell Viability assay kit was obtained from Promega (Madison, WI, USA). EGS was obtained from Pierce. Dulbecco's modified Eagle's medium (DMEM) and the supplements, fetal calf serum, L-glutamine and penicillin-streptomycin, were purchased from Biological Industries (Beit-Haemek, Israel). Anti-VDAC1 (31HL) mouse monoclonal antibodies were from Cal-Biochem (Nottingham, UK). Anti-actin monoclonal antibodies were from Millipore (Billerica, MA, USA). Monoclonal anti-CD5/CD19 and monoclonal anti-Cyto $C$ antibodies were obtained from BD Bioscience (San Jose, CA, USA). Anti-HK-I antibodies, Anti-VDAC1 (ab15895) and Cy 2- and Cy 3-conjugated anti-mouse and anti-rabbit antibodies, respectively, were obtained from Abcam. Horseradish peroxidase (HRP)-conjugated anti-mouse, anti-rabbit and anti-goat antibodies were from Promega.

Patients. Fifty-one CLL patients ( 26 women and 25 men) with a median age of 66 years were included in this study. CLL diagnosis was based on clinical examination, peripheral blood count and immuno-phenotyping, that is, co-expression of the T-cell and B-cell antigens CD5 and CD19, respectively (Supplementary Table 1). Patients received no treatment for the disease. Written informed consents were obtained from all patients, prior to sample collection, according to the Declaration of Helsinki. The ethic approval for our study was obtained from the local ethic committee of the research and was approved by the Soroka university medical center Advisory Committee on Ethics in Human Experimentation.

Peptides. The following peptides were used in this study, with the cellpenetrating sequence in bold and the amino acids added to form a loop-shaped tryptophan zipper underlined:

Antp-LP4,RQIKIWFQNRRMKWKKSWTWE-199-KKLETAVNLAWTAGNSN216KWTWK(43 residues); Tf-LP4, HAIYPRHSWTWE-199-KKLETAVNLAWTAGNSN216-KWTWK (34 residues) and N-Ter-Antp, 1-MAVPPTYADLGKSARDVFTKGYG FGL-26-RQIKIWFQNRRMKWKK (42 residues). These and other peptides used in this study were synthesized by GL Biochem (Shanghai, China) to $>85 \%$ purity and are listed in Supplementary Table 2. The peptides were dissolved in DMSO and concentrations were determined using absorbance at $280 \mathrm{~nm}$ and the specific molar excitation coefficient, as calculated based on amino acid composition.

Isolation of PBMCs and cell culture. PBMCs were isolated from venous blood of CLL patients and healthy donors by Ficoll-Paque PLUS (GE Healthcare, Buckinghamshire, UK) density gradient centrifugation, as follows. After informed consent, venous blood (10-20 ml) was drawn from CLL patients with satisfying diagnostic criteria for CLL or from normal adult donors. Blood was collected into heparin tubes and was diluted $1: 1$ with balance solution composed of two stock solutions, A (1\% D-glucose, $50 \mathrm{mM} \mathrm{CaCl}, 0.98 \mathrm{mM} \mathrm{MgCl}, 5.4 \mathrm{mM} \mathrm{KCl}$ and $0.145 \mathrm{M}$ Tris- $\mathrm{HCl}, \mathrm{pH} 7.6)$ and $\mathrm{B}(0.14 \mathrm{M} \mathrm{NaCl})$ in a $1: 9$ ratio. The resulting mix was carefully layered on Ficoll-Paque Plus ( $10 \mathrm{ml}$ of diluted blood: $15 \mathrm{ml}$ Ficoll) in 
$50 \mathrm{ml}$ conical tubes. The tubes were centrifuged at $400 \times \mathrm{g}$ (with minimal acceleration and deceleration) and $18-20^{\circ} \mathrm{C}$ for $40 \mathrm{~min}$. The fine layer of mononuclear cells was transferred to a new centrifuge tube, washed three times with balance solution and resuspended in culture medium appropriate to the application. Cells were counted by a Countess Automated Cell Counter (Invitrogen, Grand Island, NY, USA). PBMCs were used either freshly or after cryo-preservation in liquid nitrogen in 90\% FCS and 10\% DMSO $\left(2.5 \times 10^{7}\right.$ cells/ $\mathrm{ml})$. Thawed PBMCs were maintained in DMEM supplemented with the $10 \%$ fetal calf serum, $2 \mathrm{mM} \mathrm{L-glutamine,} 100 \mathrm{U} / \mathrm{ml}$ penicillin, $100 \mu \mathrm{g} / \mathrm{ml}$ streptomycin, $1 \mathrm{mM}$ sodium pyruvate, non-essential amino acids, $10 \mathrm{mM}$ Hepes and $11 \mu \mathrm{M} \beta$ mercaptoethanol (all from Biological Industries). Similar results were obtained with fresh and cryo-preserved cells. The proportion of cancerous B cells out of the total PBMC pool was analyzed by detection of CD19/CD5 double positive cells in a flow cytometer (Beckton-Dickinson, San Jose, CA, USA) using specific antibodies. MEC-1 cells were grown in DMEM + supplements and maintained in a humidified atmosphere with $5 \% \mathrm{CO}_{2}$ at $37^{\circ} \mathrm{C}$.

Determination of cell viability. Cell viability was analyzed by assaying trypan blue $(0.2 \%)$ exclusion, as counted by a Countess Automated Cell Counter (Invitrogen).

Cell treatment with VDAC1-based peptides and cell death analysis. PBMCs $\left(4 \times 10^{5}\right.$ or $8 \times 10^{5}$ cells/sample) were incubated in $200 \mu \mathrm{l}$ serum-free medium with the peptide for $90 \mathrm{~min}$ at room temperature and centrifuged $(500 \times g, 5 \mathrm{~min})$. Cell death was analyzed by PI staining and flow cytometer (Beckton-Dickinson) and BD Cell Quest Pro software. Apoptotic cell death was also analyzed by PI and annexin V-FITC staining, and by acridine orange and ethidium bromide staining ${ }^{12}$ and confocal microscopy (Olympus $1 \times 81$ ).

Morphological analysis of apoptosis by EM. For transmission EM, MEC-1 cells $\left(2.4 \times 10^{6}\right.$ cells $\left./ \mathrm{ml}\right)$ were incubated with 1 or $3 \mu \mathrm{M}$ of Antp-LP4 peptide or DMSO (final concentration of $0.1 \%$ ) for $90 \mathrm{~min}$ in serum-free medium. Cells were pelleted and fixed in Karnovsky's fixative ( $2 \%$ paraformaldehyde, $2.5 \%$ glutaraldehyde in $0.1 \mathrm{M}$ cacodylate buffer, $\mathrm{pH} 7.4$ ) for $10 \mathrm{~min}$ at room temperature and then in a fresh fixative for 3.5 days in the cold. Following buffer rinses, the cells were incubated with $1 \%$ osmium tetroxide in $0.1 \mathrm{M}$ cacodylate buffer, dehydrated in an ethanol series and epoxy propane and embedded in Araldite resin. Ultrathin sections were cut using Ultracut UCT (Leica, Vienna, Austria), mounted on formvar-coated copper grids, doubly stained with uranyl acetate and lead citrate and viewed in JEM-1230 Transmission Electron Microscope (JEOL, Tokyo, Japan). Digital images are collected with a Gatan model 830 ORIUS SC200 CCD camera using Gatan Digital Micrograph (DM) software.

ATP quantification. PBMCs were seeded in 96-well plates $\left(2 \times 10^{6} / \mathrm{ml}\right)$, incubated with the Antp-LP4 peptide, washed twice with PBS and incubated with the CellTiter-Glo reagent (Promega). Luminescence was recorded using an Infinite M1000 microplate reader (Tecan Trading, Mannedorf, Switzerland).

Mitochondrial membrane potential analysis. PBMCs were incubated with Antp-LP4 peptide, washed with PBS, incubated with TMRM (200 nM, $20 \mathrm{~min})$, washed with PBS and subjected to FACS analysis. FCCP, allowing for $\triangle \Psi$ dissipation, served as a control.

Cytochrome $\boldsymbol{c}$ and HK immunostaining. MEC-1 cells were treated with Antp-LP4 peptide, washed, paraformaldehyde-fixed (4\%, $15 \mathrm{~min})$, permeabilized with ethanol $\left(10 \mathrm{~min},-20^{\circ} \mathrm{C}\right)$, incubated with anti-Cyto $c$ or anti-HK-I antibodies and with Cy2- or Сy3-conjugated antibodies, respectively. Samples were imaged by confocal microscopy.

Cytochrome $c$ release assayed by immunoblot analysis. Release of Cyto $c$ from mitochondria to the cytosol was analyzed by immunoblot. MEC-1 cells were exposed to Antp-LP4 peptide (1 or $2 \mu \mathrm{M}$ ), harvested, washed with PBS and gently resuspended in ice-cold buffer $(100 \mathrm{mM} \mathrm{KCl}, 2.5 \mathrm{mM} \mathrm{MgCl}, 250 \mathrm{mM}$ sucrose, $20 \mathrm{mM}$ HEPES/KOH, pH 7.5, $0.2 \mathrm{mM}$ EDTA, $1 \mu \mathrm{g} / \mathrm{ml}$ leupeptin, $5 \mu \mathrm{g} / \mathrm{ml}$ cytochalasin B and $0.1 \mathrm{mM}$ PMSF) containing 0.002 or $0.035 \%$ digitonin and incubated $10 \mathrm{~min}$ on ice. Samples were centrifuged at $12000 \mathrm{~g}$ at $4^{\circ} \mathrm{C}$ for $10 \mathrm{~min}$ to obtain the supernatants (cytosolic extracts free of mitochondria) and analyzed by SDS-PAGE and immune-probed using anti-Cyto $c$ antibodies $(1: 2000$, Cat no. 556433), and then with secondary HRP-conjugated anti-mouse antibodies.
VDAC1 oligomerization and chemical cross-linking. MEC-1 cells were exposed to Antp-LP4 peptide, collected by centrifugation, re-suspended in PBS, pH 8.3, incubated at $30^{\circ} \mathrm{C}$ for 15 min with EGS $(20-50 \mu \mathrm{M})$ and subjected to SDS-PAGE and immunoblotting using anti-VDAC1 antibodies. Densitometric quantification of band intensity was performed using Multi-Gauge software (Fujifilm, Tokyo, Japan).

Gel electrophoresis and immunostaining. PBMC samples $(10-40 \mu \mathrm{g}$ protein) were subjected to SDS-PAGE and immunoblotting. Band intensities were analyzed by densitometry as above, with values normalized to the intensities of $\beta$-actin.

Statistics. Means \pm S.E. of results obtained from independent experiments are presented. The non-parametric Mann-Whitney $U$ test was used to compare control and experimental groups. A difference was considered statistically significant when the $P$-value was deemed $<0.05\left(^{*}\right),<0.01\left(^{* *}\right)$ or $<0.001\left(^{* * *}\right)$.

\section{Conflict of Interest}

The authors declare no conflict of interest.

Acknowledgements. This research was supported by a grant from the Leukemia \& Lymphoma Society. We thank Liron Dangor for assisting with the confocal microscopy and Rina Yeger for the EM work.

1. Montserrat E, Moreno C. Chronic lymphocytic leukaemia: a short overview. Ann Oncol 2008; 19(Suppl 7): vii320-vii325.

2. Byrd JC, Stilgenbauer S, Flinn IW. Chronic lymphocytic leukemia. Hematology Am Soc Hematol Educ Program 2004; 1: 163-183.

3. Bannerji R, Kitada S, Flinn IW, Pearson M, Young D, Reed JC et al. Apoptotic-regulatory and complement-protecting protein expression in chronic lymphocytic leukemia: relationship to in vivo rituximab resistance. J Clin Oncol 2003; 21: 1466-1471.

4. Gottardi D, Alfarano A, De Leo AM, Stacchini A, Aragno M, Rigo A et al. In leukaemic $\mathrm{CD} 5+\mathrm{B}$ cells the expression of $\mathrm{BCL}-2$ gene family is shifted toward protection from apoptosis. Br J Haematol 1996; 94: 612-618.

5. Schmitt B, Wendtner CM, Bergmann M, Busch R, Franke A, Pasold R et al. Fludarabine combination therapy for the treatment of chronic lymphocytic leukemia. Clin Lymphoma 2002; 3: 26-35.

6. Christian BA, Lin TS. Antibody therapy for chronic lymphocytic leukemia. Semin Hematol 2008; 45: 95-103.

7. Hanahan D, Weinberg RA. Hallmarks of cancer: the next generation. Cell 2011; 144: 646-674.

8. Mathupala SP, Ko YH, Pedersen PL. Hexokinase-2 bound to mitochondria: cancer's stygian link to the 'Warburg Effect' and a pivotal target for effective therapy. Semin Cancer Biol 2009; 19: 17-24.

9. Osterborg A, Dyer MJ, Bunjes D, Pangalis GA, Bastion Y, Catovsky D et al. Phase II multicenter study of human CD52 antibody in previously treated chronic lymphocytic leukemia. European Study Group of CAMPATH-1H Treatment in chronic lymphocytic leukemia. J Clin Oncol 1997; 15: 1567-1574.

10. Shoshan-Barmatz V, De Pinto V, Zweckstetter M, Raviv Z, Keinan N, Arbel N. VDAC a multi-functional mitochondrial protein regulating cell life and death. Mol Aspects Med 2010; 31: 227-285.

11. Shoshan-Barmatz V, Keinan N, Abu-Hamad S, Tyomkin D, Aram L. Apoptosis is regulated by the VDAC1 N-terminal region and by VDAC oligomerization: release of cytochrome $c$, AIF and Smac/Diablo. Biochim Biophys Acta 2010; 1797: 1281-1291.

12. Zaid H, Abu-Hamad S, Israelson A, Nathan I, Shoshan-Barmatz V. The voltage-dependent anion channel-1 modulates apoptotic cell death. Cell Death Differ 2005; 12: 751-760.

13. Abu-Hamad S, Arbel N, Calo D, Arzoine L, Israelson A, Keinan N et al. The VDAC1 $\mathrm{N}$-terminus is essential both for apoptosis and the protective effect of anti-apoptotic proteins. J Cell Sci 2009; 122(Pt 11): 1906-1916.

14. Arbel N, Ben-Hail D, Shoshan-Barmatz V. Mediation of the anti-apoptotic activity of BCLXL upon interaction with VDAC1. J Biol Chem 2012; 287: 23152-23161.

15. Arbel N, Shoshan-Barmatz V. Voltage-dependent anion channel 1-based peptides interact with bcl-2 to prevent antiapoptotic activity. J Biol Chem 2010; 285: 6053-6062.

16. Abu-Hamad S, Zaid H, Israelson A, Nahon E, Shoshan-Barmatz V. Hexokinase-I protection against apoptotic cell death is mediated via interaction with the voltagedependent anion channel-1: mapping the site of binding. J Biol Chem 2008; 283: 13482-13490.

17. Arzoine L, Zilberberg N, Ben-Romano R, Shoshan-Barmatz V. Voltage-dependent anion channel 1-based peptides interact with hexokinase to prevent its anti-apoptotic activity. J Biol Chem 2009; 284: 3946-3955. 
18. Grobholz R, Zentgraf H, Kohrmann KU, Bleyl U. Bax Bcl-2, fas and Fas-L antigen expression in human seminoma: correlation with the apoptotic index. Apmis 2002; 110: 724-732.

19. Schimmer AD, Munk-Pedersen I, Minden MD, Reed JC. Bcl-2 and apoptosis in chronic lymphocytic leukemia. Curr Treat Options Oncol 2003; 4: 211-218.

20. Abu-Hamad S, Sivan S, Shoshan-Barmatz V. The expression level of the voltagedependent anion channel controls life and death of the cell. Proc Natl Acad Sci USA 2006; 103: $5787-5792$.

21. Cochran AG, Tong RT, Starovasnik MA, Park EJ, McDowell RS, Theaker JE et al A minimal peptide scaffold for beta-turn display: optimizing a strand position in disulfidecyclized beta-hairpins. J Am Chem Soc 2001; 123: 625-632.

22. Stacchini A, Aragno M, Vallario A, Alfarano A, Circosta P, Gottardi D et al. MEC1 and MEC2: two new cell lines derived from B-chronic lymphocytic leukaemia in prolymphocytoid transformation. Leuk Res 1999; 23: 127-136.

23. Admoni L, Shteinfer A, Prezma T, Arbel N, Melkov A et al. VDAC1-based peptides induce selective death of B-chronic lymphocytic leukemia cells. Submitted.

24. Keinan N, Tyomkin D, Shoshan-Barmatz V. Oligomerization of the mitochondrial protein voltage-dependent anion channel is coupled to the induction of apoptosis. Mol Cell Biol 2010; 30: 5698-5709.

25. Shoshan-Barmatz V, Mizrachi D. VDAC1: from structure to cancer therapy. Front Oncol 2012; 2: 164

26. Ferraro E, Pulicati A, Cencioni MT, Cozzolino M, Francesca Navoni F, di Martino S et al. Apoptosome-deficient cells lose cytochrome $c$ through proteasomal degradation but survive by autophagy-dependent glycolysis. Mol Biol Cell 2008; 19: 3576-3588.

27. Daniels TR, Bernabeu E, Rodriguez JA, Patel S, Kozman M, Chiappetta DA et al. The transferrin receptor and the targeted delivery of therapeutic agents against cancer. Biochim Biophys Acta 2012; 1820: 291-317.

28. Smilevska T, Stamatopoulos K, Samara M, Belessi C, Tsompanakou A, Paterakis G et al. Transferrin receptor-1 and 2 expression in chronic lymphocytic leukemia. Leuk Res 2006; 30: $183-189$.

29. Brooks H, Lebleu B, Vives E. Tat peptide-mediated cellular delivery: back to basics Adv Drug Deliv Rev 2005; 57: 559-577.

30. Moede T, Leibiger B, Pour HG, Berggren P, Leibiger IB. Identification of a nuclear localization signal, RRMKWKK, in the homeodomain transcription factor PDX-1. FEBS Lett 1999; 461: 229-234.

31. Cymer F, Veerappan A, Schneider D. Transmembrane helix-helix interactions are modulated by the sequence context and by lipid bilayer properties. Biochim Biophys Acta 1818 963-973.

32. Ospina A, Lagunas-Martinez A, Pardo J, Carrodeguas JA. Protein oligomerization mediated by the transmembrane carboxyl terminal domain of Bcl-XL. FEBS Lett 2011; 585: 2935-2942.

33. Lee JH, Engler JA, Collawn JF, Moore BA. Receptor mediated uptake of peptides that bind the human transferrin receptor. Eur J Biochem 2001; 268: 2004-2012.

34. Han L, Huang R, Li J, Liu S, Huang S, Jiang C. Plasmid pORF-hTRAIL and doxorubicin co-delivery targeting to tumor using peptide-conjugated polyamidoamine dendrimer Biomaterials 2011; 32: 1242-1252.

35. Han L, Li J, Huang S, Huang R, Liu S, Hu X et al. Peptide-conjugated polyamidoamine dendrimer as a nanoscale tumor-targeted T1 magnetic resonance imaging contrast agent. Biomaterials 2011; 32: 2989-2998.

36. Oh S, Kim BJ, Singh NP, Lai H, Sasaki T. Synthesis and anti-cancer activity of covalent conjugates of artemisinin and a transferrin-receptor targeting peptide. Cancer Lett 2009; 274: 33-39.

37. Ferreira LM. Cancer metabolism: the Warburg effect today. Exp Mol Pathol 2010; 89: 372-380

38. Kaelin WG Jr., Thompson CB. Q\&A: Cancer: clues from cell metabolism. Nature 2010; 465: 562-564.
39. Mathupala SP, Ko YH, Pedersen PL., Hexokinase II. Cancer's double-edged sword acting as both facilitator and gatekeeper of malignancy when bound to mitochondria. Oncogene 2006; 25: 4777-4786

40. Wenner CE. Cell signaling and cancer-possible targets for therapy. J Cell Physiol 2010; 223: 299-308.

41. Shulga N, Wilson-Smith R, Pastorino JG. Hexokinase II detachment from the mitochondria potentiates cisplatin induced cytotoxicity through a caspase-2 dependent mechanism. Cell Cycle 2009; 8: 3355-3364.

42. Kraaijenhagen RJ, de Gast GC, van der Heiiden MC, Streefkerk M, Gmelig-Meyling FH, Rijksen $G$ et al. Isozyme distribution of hexokinase, phosphofructokinase and pyruvate kinase in lymphocytes from patients with chronic lymphocytic leukemia. Clin Chim Acta 1982; 124: 91-101.

43. Azoulay-Zohar H, Israelson A, Abu-Hamad S, Shoshan-Barmatz V. In self-defence: hexokinase promotes voltage-dependent anion channel closure and prevents mitochondria-mediated apoptotic cell death. Biochem J 2004; 377(Pt 2): 347-355.

44. Keating MJ, O'Brien S, Albitar M, Lerner S, Plunkett W, Giles F et al. Early results of a chemoimmunotherapy regimen of fludarabine, cyclophosphamide, and rituximab as initial therapy for chronic lymphocytic leukemia. J Clin Oncol 2005; 23 4079-4088.

45. Hillmen P, Skotnicki AB, Robak T, Jaksic B, Dmoszynska A, Wu J et al. Alemtuzumab compared with chlorambucil as first-line therapy for chronic lymphocytic leukemia. J Clin Oncol 2007; 25: 5616-5623.

46. Bowen DA, Call TG, Jenkins GD, Zent CS, Schwager SM, Van Dyke DL et al. Methylprednisolone-rituximab is an effective salvage therapy for patients with relapsed chronic lymphocytic leukemia including those with unfavorable cytogenetic features. Leuk Lymphoma 2007; 48: 2412-2417.

47. Castro JE, Sandoval-Sus JD, Bole J, Rassenti L, Kipps TJ. Rituximab in combination with high-dose methylprednisolone for the treatment of fludarabine refractory high-risk chronic lymphocytic leukemia. Leukemia 2008; 22: 2048-2053.

48. Advani PP, Paulus A, Masood A, Sher T, Chanan-Khan A. Pharmacokinetic evaluation of oblimersen sodium for the treatment of chronic lymphocytic leukemia. Expert Opin Drug Metab Toxicol 2011; 7: 765-774.

49. Tan W, Loke YH, Stein CA, Miller P, Colombini M. Phosphorothioate oligonucleotides block the VDAC channel. Biophys J 2007; 93: 1184-1191.

50. Vogler M, Butterworth M, Majid A, Walewska RJ, Sun XM, Dyer MJ et al. Concurrent up-regulation of $B C L-X L$ and $B C L 2 A 1$ induces approximately 1000 -fold resistance to ABT-737 in chronic lymphocytic leukemia. Blood 2009; 113: 4403-4413.

51. Merino D, Khaw SL, Glaser SP, Anderson DJ, Belmont LD, Wong C et al. Bcl-2, Bcl-x(L), and $\mathrm{Bcl}-\mathrm{w}$ are not equivalent targets of $\mathrm{ABT}-737$ and navitoclax (ABT-263) in lymphoid and leukemic cells. Blood 2012; 119: 5807-5816.

52. Raucher D, Moktan S, Massodi I, Bidwell GL 3rd. Therapeutic peptides for cancer therapy. Part II - cell cycle inhibitory peptides and apoptosis-inducing peptides. Expert Opin Drug Deliv 2009; 6: 1049-1064

53. Kaspar AA, Reichert JM. Future directions for peptide therapeutics development. Drug Discov Today May 2013; S1359-6446: 00157-00158.

(c) (1) Cell Death and Disease is an open-access journal che licensed under a Creative Commons Attribution-NonCommercialShareAlike 3.0 Unported License. To view a copy of this license, visit http://creativecommons.org/licenses/by-nc-sa/3.0/ 